\title{
Multifractal analysis of vertical total electron content (VTEC) at equatorial region and low latitude, during low solar activity
}

\author{
M. J. A. Bolzan ${ }^{1,2}$, A. Tardelli ${ }^{2}$, V. G. Pillat ${ }^{2}$, P. R. Fagundes ${ }^{2}$, and R. R. Rosa ${ }^{3}$ \\ ${ }^{1}$ Laboratório de Física, Universidade Federal de Goiás (UFG), Jataí, GO, Brazil \\ ${ }^{2}$ Laboratório de Física e Astronomia, IP\&D, Universidade do Vale do Paraíba (UNIVAP), São José dos Campos, SP, Brazil \\ ${ }^{3}$ Laboratório Associado de Computação e Matemática Aplicada (LAC), Instituto Nacional de Pesquisas Espaciais (INPE), \\ São José dos Campos, SP, Brazil
}

Correspondence to: M. J. A. Bolzan (mauricio.bolzam@pq.cnpq.br)

Received: 29 May 2012 - Revised: 18 December 2012 - Accepted: 21 December 2012 - Published: 28 January 2013

\begin{abstract}
This paper analyses the multifractal aspects of the GPS data (measured during a period of low solar activity) obtained from two Brazilian stations: Belém $\left(01.3^{\circ} \mathrm{S}, 48.3^{\circ} \mathrm{W}\right)$ and São José dos Campos (SJC) $\left(23.2^{\circ} \mathrm{S}, 45.9^{\circ} \mathrm{W}\right)$. The results show that the respective geographic sites show important scaling differences as well as similarities when their multifractal signatures for vertical total electron content (VTEC) are compared. The $f(\alpha)$ spectra have a narrow shape for great scales, which indicates the predominance of deterministic phenomena, such as solar rotation (27 days) over intermittent phenomena. Furthermore, the $f(\alpha)$ spectra for both sites have a strong multifractality degree at small scales. This strong multifractality degree observed at small scales (1 to $12 \mathrm{~h}$ ) at both sites is because the ionosphere over Brazil is a non-equilibrium system. The differences found were that Belém presented a stronger multifractality at small scales $(1 \mathrm{~h}$ to $12 \mathrm{~h}$ ) compared with SJC, particularly in 2006 . The reason for this behaviour may be associated with the location of Belém, near the geomagnetic equator, where at this location the actions of X-rays, ultraviolet, and another wavelength from the Sun are more direct, strong, and constant throughout the whole year. Although the SJC site is near ionospheric equatorial anomaly (IEA) peaks, this interpretation could explain the higher values found for the intermittent parameter $\mu$ for Belém compared with SJC. Belém also showed the presence of one or two flattening regions for $f(\alpha)$ spectra at the same scales mentioned before. These differences and similarities also were interpreted in terms of the IEA content, where this phenomenon is an important source of intermittence due the presence of the VTEC peaks at $\pm 20^{\circ}$ geomagnetic latitudes.
\end{abstract}

Keywords. Ionosphere (Equatorial ionosphere)

\section{Introduction}

Studying the relations between solar and geomagnetic/ionospheric phenomena on the Earth is important due to their consequences on human activities (Bolzan et al., 2009b; Bolzan and Rosa, 2012). Recently, global position system (GPS) satellites have been used to study the effects of solar disturbances on earth's geomagnetic field variations (Krankowski et al., 2005).

According to previous works (Bolzan et al., 2009a, b; Bolzan and Rosa, 2012), the vertical total electron content (VTEC) time series have very intermittent characteristics, mainly at small scales. In addition, the VTEC time series depend on geomagnetic substorm and storm activities. The two distinct observations of a global self-organized state (SO) and a repetitive state promoted by substorm cycle (Valdivia et al., 2005) show that the geomagnetic system is complex. According to Bolzan et al. (2009b), these problems have external origin due to the Sun-Earth interactions (Gonzales et al., 2002) along with internal origins such as gravity and planetary waves (Fagundes et al., 2005, 2009a, b; Bolzan et al., 2009b). Thus, the action of these physical phenomena makes the VTEC time series strongly intermittent. This fact shows that it is important to use a statistical methodology to discover some universal characteristics of the VTEC at both studied sites.

Recently, Bolzan et al. (2009b) examined the probability density functions (PDFs) in VTEC time series obtained in 


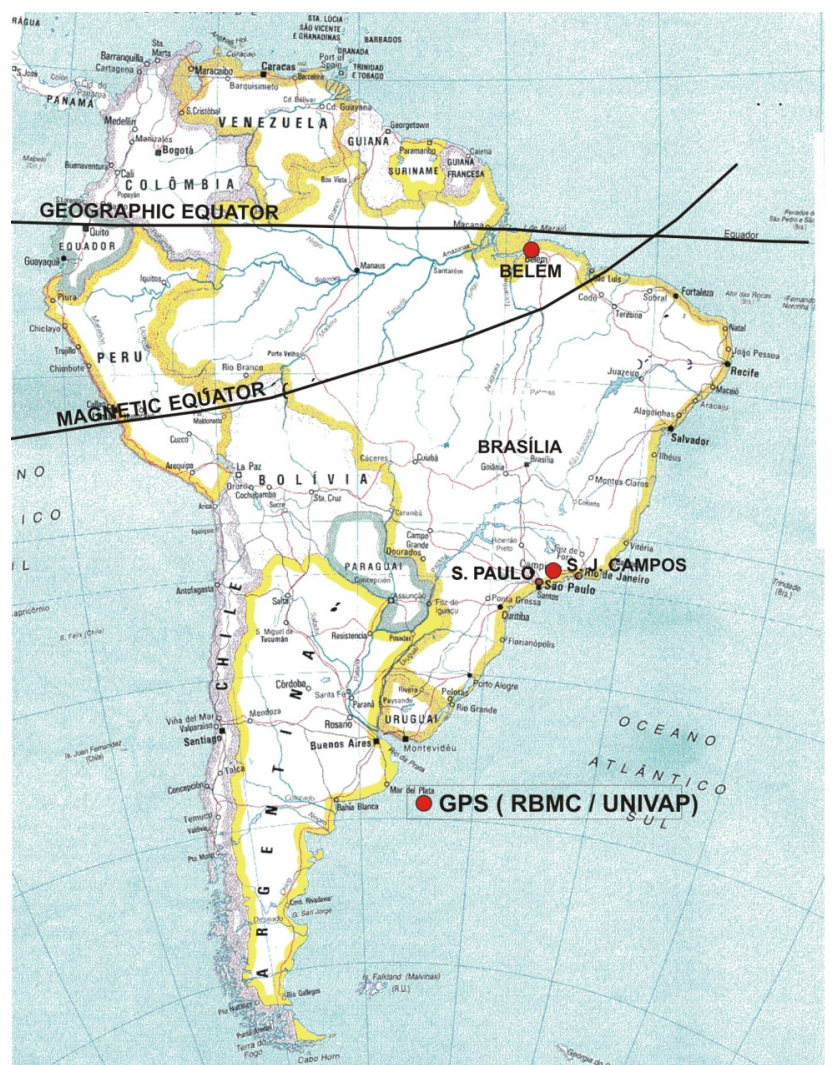

Fig. 1. Map of the GPS ground facilities in the Brazilian cities of São José dos Campos and Belém.

Brazil, for the first time. Their results show that, at the small scale $(\sim 3 \mathrm{~min})$, the PDFs had a very stretched exponential form, where this shape is associated with intermittent phenomena due to turbulence (e.g. Bolzan et al., 2005).

As mentioned by Rosa et al. (2009), the intermittency leads to deviation from usual Kolmogorov energy structure functions and its main signature is the singularity spectra exponents, $f(\alpha)$, which represent a power-law scalingfree dependence (Frisch, 1995). Recently, Pont et al. (2009) searched for a common multifractal signature in six different systems. According to them, these six systems had the same shape of the singularity spectra exponents, $f(\alpha)$, after using the reduced singularity spectrum. The objective of this work is to discover the possible differences of the $f(\alpha)$ from two Brazilian sites (equatorial region and low latitude), without using the approach of Pont et al. (2009) to obtain the common multifractal signatures from the two sites. Our approach is based on four data sets, and at least one relevant aspect is addressed in this paper, which is the comparative study for two Brazilian sites based on the multifractal characteristic at different time scales.
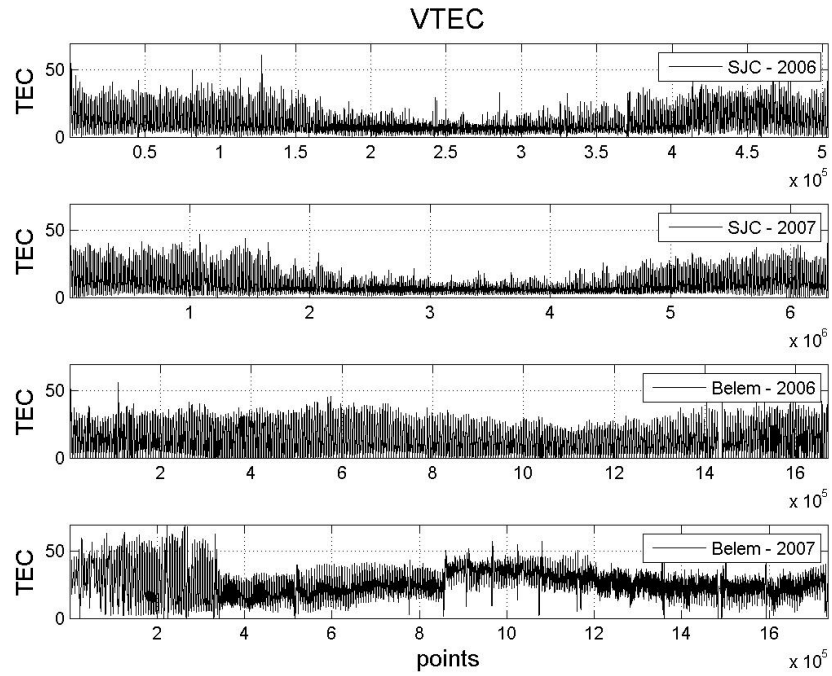

Fig. 2. The four time series shown in legend.

\section{Data and methodology}

\subsection{Data}

This work used data obtained through GPS receivers at two Brazilians sites: São José dos Campos (SJC, low latitude) and Belém (BE equatorial region). These sites are located at different geomagnetic hemispheres. Figure 1 is a map showing the two GPS receiver facilities. The annual time series of the VTEC was obtained at São José dos Campos using the GPS monitor (model Leica 1200) in operation at Universidade do Vale do Paraíba (Bolzan et al., 2009b). Furthermore, the annual time series of the TEC (total electron content) data sets were obtained for Belém using data from the Brazilian Continuous Monitoring Network (RBMC) through the website http://www.ibge.gov.br/home/geociencias/geodesia/ rbmc/rbmc.shtm?c=7. Figure 2 shows the four time series used in this work.

TEC is the total number of electrons present along any path between the ground stations and GPS, with units of electrons per square meter, where $10^{16}$ electrons $\mathrm{m}^{-2}=1 \mathrm{TEC}$ unit (TECU). According to Adewale et al. (2012), the VTEC is derived from slant TEC (STEC) by using the following equation:

$\mathrm{VTEC}=\left[\mathrm{STEC}-\left(b_{\mathrm{R}}+b_{\mathrm{S}}\right)\right] / S(E)$,

where $b_{\mathrm{R}}$ is the inter-frequency differential receiver biases and $b_{\mathrm{S}}$ the inter-frequency differential satellite biases. The mapping function $S(E)$ employed is given by

$S(E)=\frac{1}{\cos (Z)}=\left\{1-\left(\frac{R_{\mathrm{E}} \times \cos (E)}{R_{\mathrm{E}}+h_{\mathrm{s}}}\right)^{2}\right\}^{-0.5}$

with $z=$ zenith angle of the satellite as seen from the observing station, $R_{\mathrm{E}}=$ radius of the Earth, $E=$ the elevation angle 
in radians, and $h_{\mathrm{s}}=$ the altitude of the thin layer above the surface of the Earth (taken as $350 \mathrm{~km}$ ).

The power spectrum for the VTEC time series displayed a sizeable scaling range with a slope of approximately $-5 / 3$, as shown in Fig. 3. Despite this behaviour in the VTEC time series, all time series are rich in intermittency for both sites.

\subsection{Multifractal analysis}

To obtain the singularity spectrum $f(\alpha)$ from the VTEC data sets, we used the wavelet transform modulus maxima (WTMM) (Muzy et al., 1991). The WTMM method provided the partition function over the modulus maxima of the wavelet transform (WT) of a $\operatorname{VTEC}(t)$ time series (Rosa et al., 2009).

The WT of the $\operatorname{VTEC}(t)$ time series is given by

$$
W_{\psi}[\operatorname{VTEC}(t)](a, b)=\frac{1}{\sqrt{a}} \int_{-\infty}^{\infty} H(t) \psi^{*}((t-b) / a) d t
$$

where $H(t)$ is the time series of the VTEC, $\psi^{*}$ the complex conjugate of a continuous wavelet function $\psi, a>0$ the scaling factor controlling the dilation of the mother wavelet, and $b$ the translation parameter determining the shift of the mother wavelet. It is common to use the Morlet wavelet function given by (Farge, 1992)

$\psi(t)=\pi^{-1 / 4} e^{i \omega_{0} t} e^{-t^{2} / 2}$

where $\omega_{0}$ is the frequency parameter that allows one to shift the frequency range for investigations, but we used a fixed value at $\omega_{0}=6$.

The wavelet transform can reveal the local characteristics of $\operatorname{VTEC}(t)$ at a point $t_{0}$. More precisely, we have the following power-law relation:

$W_{\psi}[\operatorname{VTEC}(t)]\left(a, t_{0}\right) \approx|a|^{\alpha\left(t_{0}\right)}$

where $\alpha\left(t_{0}\right)$ is the singularity strength, also called Hölder exponent. We obtain the exponent $\alpha\left(t_{0}\right)$, for fixed location $x_{0}$, through the log-log plot of the WT amplitude versus the scale $a$. This power-law relation is not easy to obtain when the process is given by a hierarchical distribution of singularities, making difficult the accurate acquisition of $\alpha$ on a finite range of scales. The local maxima of $W_{\psi}(a, b)$ at a given scale $\alpha$ are likely to contain all the hierarchical distribution of singularities in the signal (Muzy et al., 1991). As mentioned by Bolzan et al. (2009a), at a given scale $\alpha$, each of the WTMM bifurcates into two new maxima, giving rise to a rich multiplicative cascade in the limit $a \rightarrow 0$. Thus, it is possible to identify a space-scale partitioning over the maxima distribution, and, consequently, a usual thermodynamical method of computing the multifractal spectrum of $\operatorname{VTEC}(t)$ is to define a partition function, which scales, in the limit $a \rightarrow 0$, as (Muzy et al., 1991)

$Z(a, q)=\sum_{n}\left|W_{\psi}[\operatorname{VTEC}(t)]\left(a, t_{n}\right)\right|^{q} \approx a^{\tau(q)}$

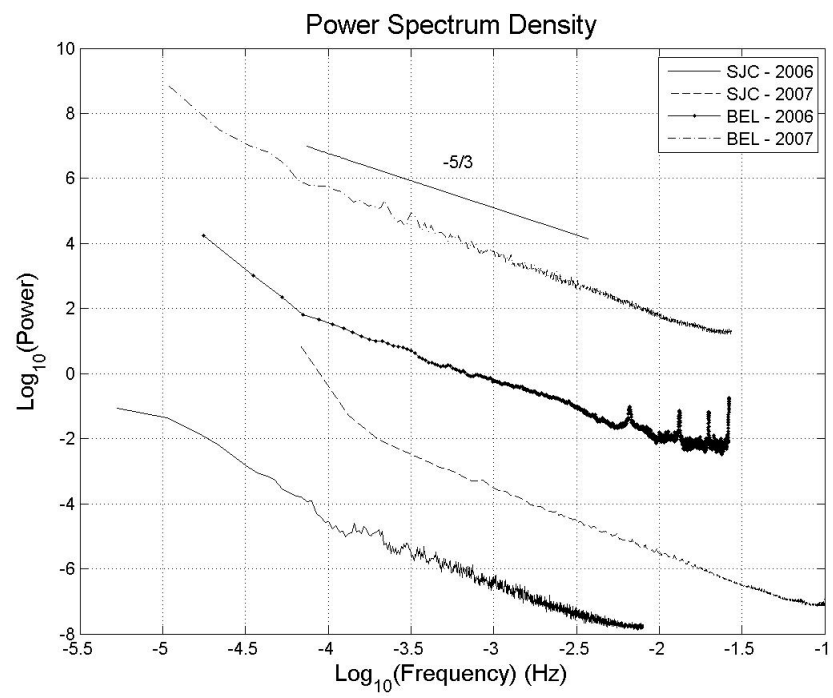

Fig. 3. Power spectral density for the time series shown in legend. The entire spectrum was vertically shifted for better visualization. The straight black line corresponds to the $-5 / 3$ slope.

where $t_{n}$ is the position of all local maxima at a fixed scale $a$, and $q$ is the moment of the measure distributed on the WTMM hierarchy used to define the power-law scaling of $Z(a, q)$. This process provides the power-law for small $\alpha$ the scaling exponents $\tau(q)$ - the multifractal spectrum (Muzy et al., 1991). Arneodo et al. (1995) used a hierarchy of the WTMM to obtain the partition function $Z(a, q)$ based on the multifractal formalism.

The analysis of the singularity spectrum, $f(\alpha)$, for a set of scales $a$, corresponds to the final step in the WTMM method used here. If a single value $\alpha$ is found for all singularities $t_{n}$, the signal has a monofractal structure; however, if we find different values of $\alpha$, then the underlying process is multifractal (Oswiecimka et al., 2006). The singularity spectrum, approximately an upside-down parabola, peaks at $f_{\alpha, \max }$. The range $\mu=\alpha_{\max }-\alpha_{\min }$ quantifies the fractal non-uniformity and is also related to the intermittence degree, while $f_{\alpha}$ characterizes how frequently burst components with scaling exponent $\alpha$ occur.

\section{Results and interpretation}

In order to compute the characteristic $f(\alpha)$ for VTEC from both Brazilian sites, we chose a set of time scales characteristic in the $\operatorname{VTEC}(t)$ time series to use in the Morlet WT. We used the concept of scale $(r)$ through the difference $\operatorname{VTEC}(t, r)=\operatorname{VTEC}(t+r)-\operatorname{VTEC}(t)$, without removing the daily variability component. As a representative set of VTEC oscillations, we chose the following typical characteristic scales: 1 and $12 \mathrm{~h}, 1,5$, and 27 days. For the sake to simplicity, we show only the singularity spectrum, $f(\alpha)$, 

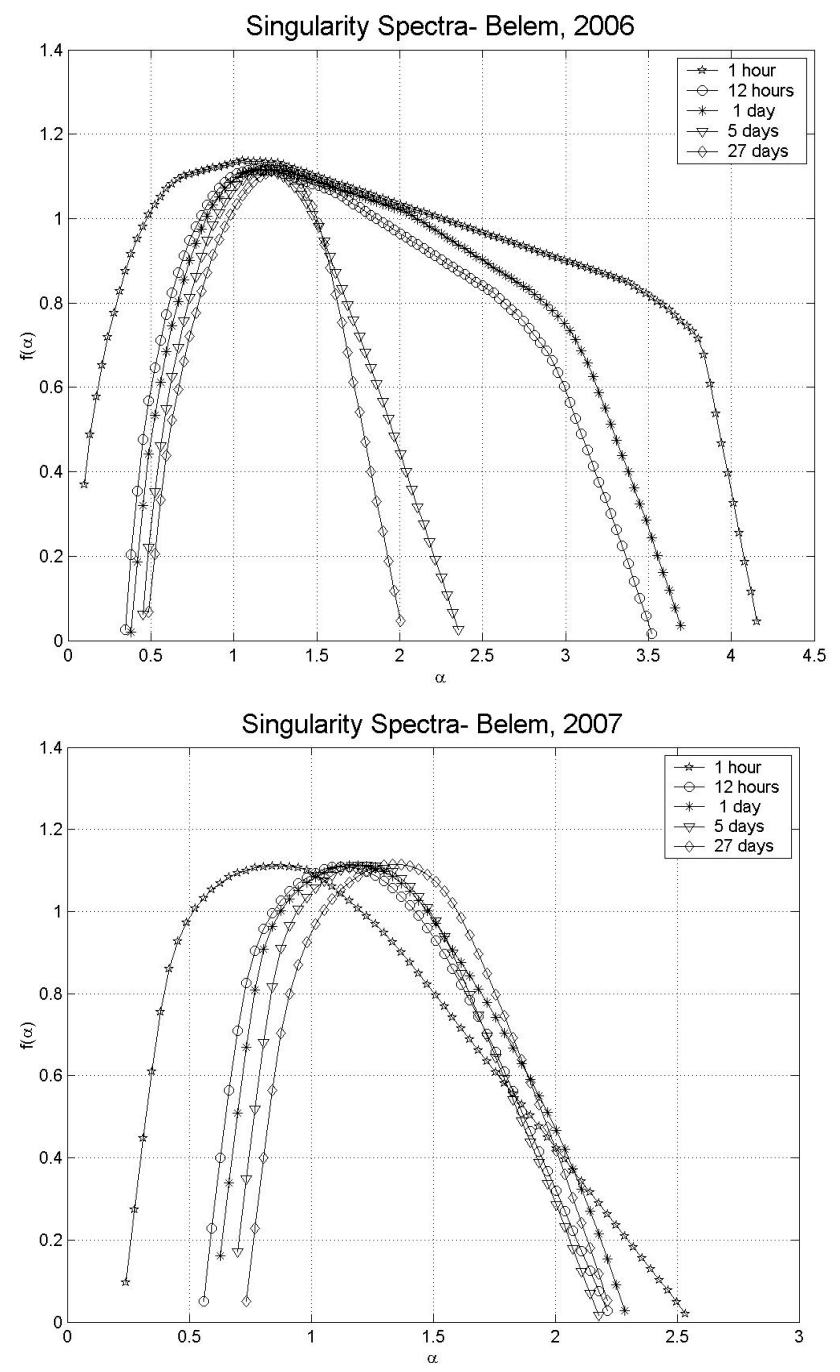

Fig. 4. Singularity spectrum for characteristic time scales shown in legend for Belém, 2006 and 2007.

characteristics for Belém and SJC for both years in Figs. 4 and 5, respectively.

For Fig. 4, the spectra $f(\alpha)$ present a well-developed Gaussian shape for all scales as well as a wide range of the $\alpha$ values, starting at zero to 4 for 2006 and zero to 2 for 2007, showing the higher multifractality degree for Belém. The $f(\alpha)$ spectrum for Belém 2006 shows a stronger multifractality degree due to the wider spectrum; i.e. the $\alpha$ values start at 0.1 and go to 3.6 at the smallest scales $(1 \mathrm{~h}, 12 \mathrm{~h}$, and $24 \mathrm{~h}$ ). Another peculiar behaviour, observed also in Belém 2006 , is indicated by one or two flattening regions for the $f(\alpha)$ spectra, from the scale of $1 \mathrm{~h}$ to 1 day. In our previous work (Bolzan et al., 2009a), we observed this behaviour for the H-component of geomagnetic field obtained in Brazil. Through simple numerical simulation, we conjectured that this behaviour may be associated with some aspects of geo-
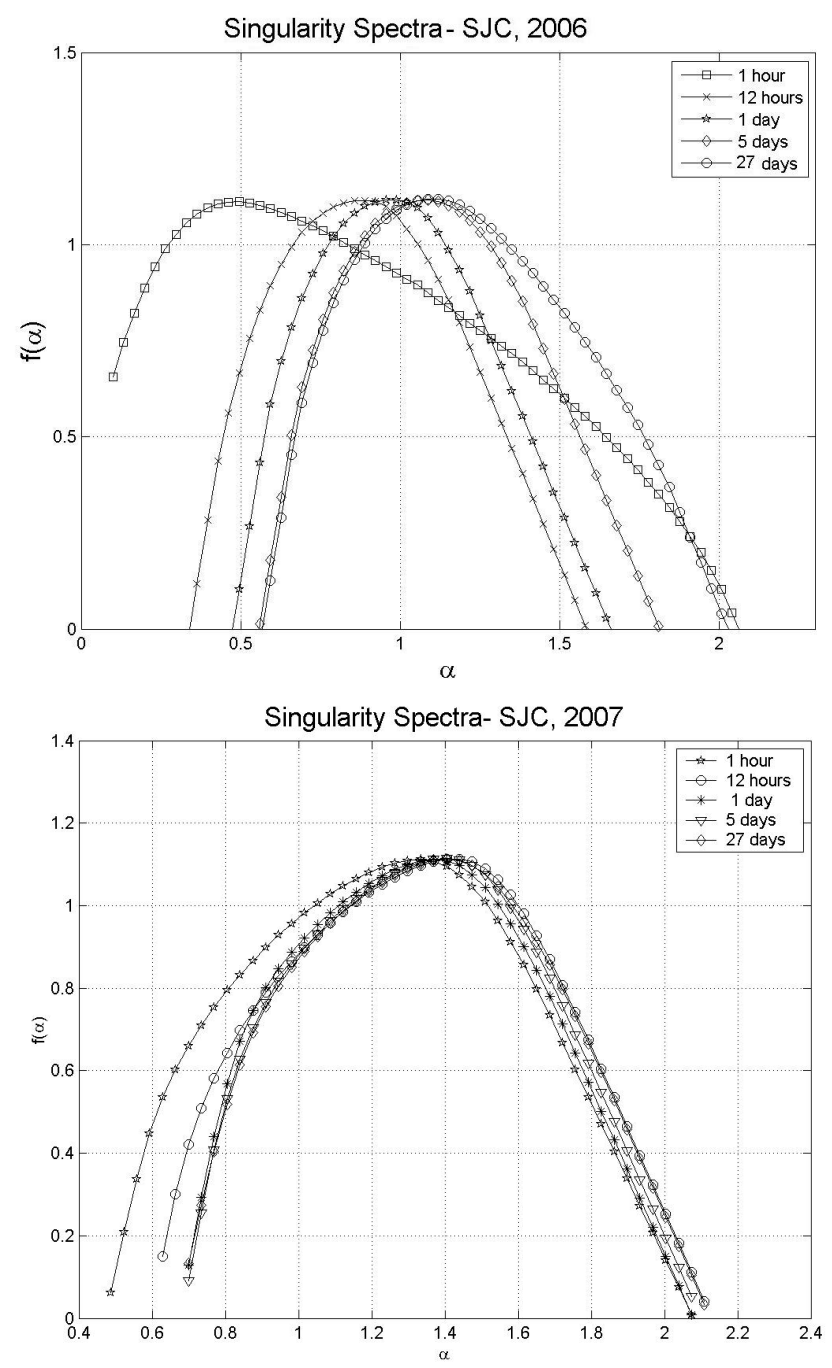

Fig. 5. Singularity spectrum for characteristic time scales shown in legend for SJC, 2006 and 2007.

magnetic substorm and storm, which are still poorly understood, causing the increase of the intermittence phenomena.

For Fig. 5, the spectra $f(\alpha)$ for SJC for both years also present a developed Gaussian shape, showing a multifractality degree for all scales. However, the spectrum for SJC is narrower; i.e. the $\alpha$ values start at 0.4 and go to 2, approximately, for all scales.

It is important to point out some differences and similarities between singularity spectra $f(\alpha)$ for SJC and Belém. As mentioned before, the presence of the flattening regions for $f(\alpha)$ spectra is an important difference in the spectra of Belém and SJC. However, similarity also exists between two sites, in that all the $f(\alpha)$ spectra from both sites have a narrow shape, indicating the predominance of the deterministic phenomena, such as solar rotation (27 days), over intermittent phenomena. 
Table 1. $\mu$ parameter for each characteristic scale for both sites and years.

\begin{tabular}{ccccc}
\hline Period & $\mu$ & $\mu$ & $\mu$ & $\mu$ \\
& $(\mathrm{BE}-2006)$ & $(\mathrm{BE}-2007)$ & $(\mathrm{SJC}-2006)$ & $(\mathrm{SJC}-2007)$ \\
\hline $1 \mathrm{~h}$ & 4.06 & 2.30 & 1.95 & 1.59 \\
$12 \mathrm{~h}$ & 3.17 & 1.66 & 1.62 & 1.48 \\
1 day & 3.31 & 1.66 & 1.14 & 1.37 \\
5 days & 1.90 & 1.48 & 1.25 & 1.37 \\
27 days & 1.52 & 1.48 & 1.41 & 1.41 \\
\hline
\end{tabular}

In addition to the possible action of the geomagnetic substorm and storm, equatorial ionospheric irregularities are present. These phenomena originate in the geomagnetic equator region and extend to low latitudes, and they may be an important source of intermittence there. The work of the de Rezende et al. (2007), using GPS data from two Brazilian sites (São Luiz and SJC), showed the generation and evolution of these phenomena over the Brazilian sector. According to them, the equatorial ionospheric irregularities (also called plasma bubbles) occur from September to March and last for about $6 \mathrm{~h}$.

For a better comparison between the intermittence degrees from both sites, we performed the parameter $\mu=\alpha_{\max }-$ $\alpha_{\min }$, which quantifies the intermittence degree, as mentioned before. Table 1 shows this parameter for both cities and for both years. There is a strong difference between Belém and SJC for both years; i.e. Belém had a higher intermittence degree than SJC. The reason for this behaviour may be associated with the location of Belém, near the geomagnetic equator. At this location, the actions of X-ray, ultraviolet, and other wavelengths from the Sun are more direct, strong, and constant throughout the year, if compared with SJC, which is situated at $23^{\circ} \mathrm{S}$. This fact is more evident when comparing the parameter $\mu$ only at small scale, i.e. period of $1 \mathrm{~h}$ (see Table 1). Walker (1981) and Walker and Strickland (1981) showed that the region between $-20^{\circ}$ and $+20^{\circ}$ of geomagnetic latitude experiences a phenomenon called ionospheric equatorial anomaly (IEA), which is an important source of intermittence due to the presence of the VTEC peaks at geomagnetic latitudes. The SJC site is near $-20^{\circ}\left(-23^{\circ}\right.$ in fact $)$ and would have strong influence from the IEA, resulting in higher values of the $\mu$ parameter compared to the Belém site. However, it is important to mention that we analysed data sets obtained from low solar activity periods, 2006 and 2007, and the IEA would not be strong compared with high solar activity period, as studied by Walker (1981) and Walker and Strickland (1981). This fact could explain the higher values found for the $\mu$ parameter for Belém compared with SJC, even though the last site is near IEA peaks.

Another important parameter can be extracted from the $f(\alpha)$ spectrum, which is given by $\alpha$, where this spectrum has a maximum value. This $\alpha$ reveals the most "frequent" singularity for all the analysed time series. Table 2 shows
Table 2. The $\alpha$ parameter for each characteristic scale for both sites and years.

\begin{tabular}{ccccc}
\hline Period & $\begin{array}{c}\alpha \\
(\mathrm{BE}-2006)\end{array}$ & $\begin{array}{c}\alpha \\
(\mathrm{BE}-2007)\end{array}$ & $\begin{array}{c}\alpha \\
(\mathrm{SJC}-2006)\end{array}$ & $\begin{array}{c}\alpha \\
(\mathrm{SJC}-2007)\end{array}$ \\
\hline $1 \mathrm{~h}$ & 1.05 & 0.88 & 0.49 & 1.33 \\
$12 \mathrm{~h}$ & 1.09 & 1.12 & 0.88 & 1.44 \\
1 day & 1.16 & 1.16 & 0.98 & 1.37 \\
5 days & 1.20 & 1.23 & 1.11 & 1.40 \\
27 days & 1.27 & 1.33 & 1.11 & 1.40 \\
\hline
\end{tabular}

this parameter obtained for the same five scales. According to Telesca et al. (2004), the $\alpha$ parameter increases toward the great scales due to the presence of the deterministic phenomena in the time series. In the present work, diurnal variation ( 1 day scale) up to full solar rotation (27 day scale) may be responsible for the reduction of the multifractality degree observed in VTEC time series, because these phenomena are strong and deterministic, as pointed out in our previous work (Bolzan et al., 2009b). This behaviour is more evident for the equatorial region (Belém) than at low latitude (SJC).

\section{Concluding remarks}

This work analysed the multifractal aspects of the GPS data obtained from two Brazilian sites, Belém and SJC, located at different geographic sites and for 2006 and 2007. The multifractal signature, characterized by a well-developed Gaussian shape on the singularity spectra, $f(\alpha)$ and obtained through use of the WTMM approach, was observed for both sites and years. Some differences and similarities between both sites were successfully detected.

An important and peculiar behaviour was observed in Belém in 2006, and was characterized by one or two flattening regions for the $f(\alpha)$ spectra, from the scale of $1 \mathrm{~h}$ to 1 day. Through our previous work (Bolzan et al., 2009a), it is possible to conjecture that this behaviour may be associated with some aspects of geomagnetic substorm and storm, which are still poorly understood, causing the increase of the intermittence phenomena. Furthermore, the flatness present only in the spectra from Belém at scales of $1 \mathrm{~h}, 12 \mathrm{~h}$, and 1 day can be associated with strong activity of gravity waves and strong component of semi-diurnal and diurnal tides at equatorial region, respectively (MacDougal et al., 2009). However, the $f(\alpha)$ spectra for the 5 and 27 days for Belém and $1 \mathrm{~h}, 12 \mathrm{~h}, 1$ day, 5 days, and 27 days for SJC do not show pronounced flatness, indicating less intermittent activities at these scales.

Results from the parameter $\mu=\alpha_{\max }-\alpha_{\min }$ show that Belém has a stronger multifractality at small scales $(1 \mathrm{~h}$ to $12 \mathrm{~h}$ ) compared with SJC. We conjecture that the reason for this behaviour may be associated with the location of Belém, near the geomagnetic equator. At this location, the actions of $\mathrm{X}$-ray, ultraviolet, and another wavelengths from the Sun are 
more direct, strong, and constant throughout the year, compared with SJC, which is situated at $23^{\circ} \mathrm{S}$. This fact could explain the higher values found for the $\mu$ parameter for Belém compared with SJC, even though the last site is near IEA peaks (Walker, 1981; Walker and Strickland, 1981).

Despite these differences, these results can be normalized to find universal characteristics, even for VTEC time series obtained in distinct locations. A common characteristic found in this work was that the $f(\alpha)$ spectra for both sites present a narrow shape for great scales, which indicates the predominance of deterministic phenomena, such as solar rotation (27 days), over intermittent phenomena; and the $f(\alpha)$ spectra for both sites have a strong multifractality degree at small scales. This strong multifractality degree observed at small scales ( 1 to $12 \mathrm{~h}$ ) at both sites is because the ionosphere over Brazil is a non-equilibrium system. According to Wanliss and Dobias (2007), a change in temporal scaling for non-equilibrium systems may be referred to as a dynamical phase transition and the ionosphere is a good example of this fact. However, despite some differences found between the sites, it is possible to characterize the common behaviour found in this work through specific parameters. Thus, our next step will be finding an approach to normalize these results for different locations with the objective of obtaining some universal parameters. This objective will permit us to model the multifractal spectral such as the p-mode (Halsey et al., 1986).

Acknowledgements. The Brazilian agencies CNPq (grant number 301457/2009-3) and FAPESP (grant number 2008/05482-5). The authors thank the reviewers for suggestions and comments, which have substantially improved the paper.

Topical Editor K. Kauristie thanks M. Gende and one anonymous referee for their help in evaluating this paper.

\section{References}

Adewale, A. O., Oyeyemi, E. O., and Olwendo, J.: Solar activity dependence of total electron content derived from GPS observations over Mbarara, Adv. Space Res., 50, 415-426, doi:10.1016/j.asr.2012.05.006, 2012.

Arneodo, A., Bacry, E., and Muzy, J. F.: The thermodynamics of fractals revisited with wavelets, Physica A, 213, 232-275, 1995.

Bolzan, M. J. A. and Rosa, R. R.: Multifractal analysis of interplanetary magnetic field obtained during CME events, Ann. Geophys., 30, 1107-1112, doi:10.5194/angeo-30-1107-2012, 2012.

Bolzan, M. J. A., Sahai, Y., Fagundes, P. R., Rosa, R. R., Ramos, F. M., and Abalde, J. R.: Intermittency analysis of geomagnetic storm time-series observed in Brazil, J. Atmos. Solar-Terr. Phys., 67, 1365-1372, doi:10.1016/j.jastp.2005.06.008, 2005.

Bolzan, M. J. A., Becker-Guedes, F., Fagundes, P. R., Sahai, Y., Pillat, V. G., and Wrasse, C. M.: Statistical analysis of the total electron content observed at 23 degrees $\mathrm{S}$ in the Brazilian sector, Adv. Space Res., 44, 385-394, doi:10.1016/j.asr.2009.03.012, 2009a.
Bolzan, M. J. A., Rosa, R. R., and Sahai, Y.: Multifractal analysis of low-latitude geomagnetic fluctuations, Ann. Geophys., 27, 569576, doi:10.5194/angeo-27-569-2009, 2009b.

de Rezende, L. F. C., de Paula, E. R. Kantor, I. J., and Kintner, P. M.: Mapping and Survey of Plasma Bubbles over Brazilian Territory, J. Navigation, 60, 69-81, doi:10.1017/S0373463307004006, 2007.

Fagundes, P. R., Pillat, V. G., Bolzan, M. J. A., Sahai, Y., BeckerGuedes, F., Abalde, J. R., Aranha, S. L., and Bittencourt, J. A.: Observations of $\mathrm{F}$ layer electron density profiles modulated by planetary wave type oscillations in the equatorial ionospheric anomaly region, J. Geophys. Res., 110, A12302, doi:10.1029/2005JA011115, 2005.

Fagundes, P. R., Abalde, J. R., Bittencourt, J. A., Sahai, Y., Francisco, R. G., Pillat, V. G., and Lima, W. L. C.: F layer postsunset height rise due to electric field prereversal enhancement: 2 . Traveling planetary wave ionospheric disturbances and their role on the generation of equatorial spread F, J. Geophys. Res., 114, A12322, doi:10.1029/2009JA014482, 2009a.

Fagundes, P. R., Bittencourt, J. A., Abalde, J. R., Sahai Y., Bolzan, M. J. A., Pillat, V. G., and Lima, W. L. C.: F layer postsunset height rise due to electric field prereversal enhancement: 1. Traveling planetary wave ionospheric disturbance effects, J. Geophys. Res., 114, A12321, doi:10.1029/2009JA014390, 2009b.

Farge, M.: Wavelet transforms and their applications to turbulence, Annu. Rev. Fluid Mechanics, 24, 395-457, 1992.

Frisch, U.: Turbulence, Cambridge University Press, New York, 1995.

Gonzalez, W. D., Tsurutani, B. T., Lepping, R. P., and Schwenn, R.: Interplanetary phenomena associated with very intense geomagnetic storms, J. Atmos. Solar-Terr. Phys., 64, 173-181, 2002.

Halsey, T. C., Jensen, M. H., Kadanoff, L. P., Procaccia, I., and Shraiman, B. I.: Fractal measures and singularities - The characterization of strange sets, Physical Review A, 33, 1141-1151, 1986.

Krankowski, A., Kosek, W., Baran, L. W., and Popinski, W.: Wavelet analysis and forecasting of VTEC obtained with GPS observations over European latitudes, J. Atmos. SolarTerr. Phys., 67, 12, 1147-1156, doi:10.1016/j.jastp.2005.03.004, 2005.

MacDougall, J., Abdu, M. A., Batista, I., Fagundes, P. R., Sahai, Y., and Jayachandran, P. T.: On the production of traveling ionospheric disturbances by atmospheric gravity waves, J. Atmos. Solar-Terr. Phys., 71, 17-18, 2013-2016, doi:10.1016/j.jastp.2009.09.006, 2009.

Muzy, J. F., Bacry, E., and Arneodo, A.: Wavelets and Multifractal formalism for singular signals: Application to turbulence data, Phys. Rev. Lett., 67, 3515-3518, 1991.

Oswiecimka, P., Kwapien, J., and Drozdz, S.: Wavelet versus detrended fluctuation analysis of multifractal structures, Phys. Rev. E, 74, 016103, doi:10.1103/PhysRevE.74.016103, 2006.

Pont, O., Turiel, A., and Pérez-Vicente, C. J.: Empirical evidences of a common multifractal signature in economic, biological and physical systems, Physica A-Statistical Mechanics and its Applications, 388, 2025-2035, doi:10.1016/j.physa.2009.01.041, 2009.

Rede Brasileira de Monitoramento Contínuo (RBMC), IBGE, available at: http://www.ibge.gov.br/home/geociencias/geodesia/ rbmc/rbmc.shtm?c=7, 2012. 
Rosa, R. R., Ramos, F. M., Caretta, C. A., and Velho, H. F. C.: Extreme event dynamics in the formation of galaxy-sized dark matter structures, Computer Physics Communications, 180, 621624, doi:10.1016/j.cpc.2008.11.018, 2009.

Telesca, L., Lapenna, V., Vallianatos, F., Makris, J., and Saltas, V.: Multifractal features in short-term time dynamics of ULF geomagnetic field measured in Crete, Greece, Chaos, Solitons \& Fractals, 21, 273-282, doi:10.1016/j.chaos.2003.10.020, 2004.

Valdivia, J. A., Rogan, J., Muñoz, V., Gomberoff, L., Klimas, A., Vassiliadis, D., Uritsky, V., Sharma, S., Toledo, B., and Wastavino, L.: The magnetosphere as a complex system, Adv. Space Res., 35, 961-971, doi:10.1016/j.asr.2005.03.144, 2005.
Walker, G. O.: Longitudinal structure of the F-region equatorial anomaly - a review, J. Atmos. Terr. Phys., 43, 763-774, 1981.

Walker, G. O. and Strickland, A. E.: A comparison of the ionospheric equatorial anomaly in the East Asian and the American regions at sunspot minimum, J. Atmos. Terr. Phys., 43, 589-595, 1981.

Wanliss, J. A. and Dobias, P.: Space storm as a phase transition, J. Atmos. Solar-Terr. Phys., 69, 675-684, doi:10.1016/j.jastp.2007.01.001, 2007. 